\title{
MHD Free and Forced Convective Flow in a Rotating Channel
}

\author{
B. C. Sarkar \\ Department of Applied Mathematics \\ Vidyasagar University \\ Midnapore 721 102, India
}

\author{
S. Das \\ Department of Mathematics \\ University of Gour Banga \\ Malda 732 103, India
}

\author{
R. N. Jana \\ Department of Applied Mathematics \\ Vidyasagar University \\ Midnapore 721 102, India
}

\begin{abstract}
A steady MHD free and forced convective viscous incompressible electrically conducting flow in a rotating channel under constant pressure gradient has been studied. An exact solution of the governing equations has been obtained in closed form. The numerical results for the velocity components, the induced magnetic field components and the temperature distribution are being presented graphically. The shear stresses and critical Grashof numbers at the lower and upper plates have been calculated. The heat transfer characteristics have also been studied on taking viscous and Joule dissipations into account. The rate of heat transfer at the lower plate increases whereas the rate of heat transfer at the upper plate decreases with an increase in either magnetic parameter or Eckert number or Grashof number.
\end{abstract}

\section{Keywords}

MHD flow, free and forced convection, induced magnetic field, rotation, Grashof number, heat transfer and Eckert number.

\section{INTRODUCTION}

The study of hydromagnetic viscous conducting rotating flows has drawn attention due to occurrence of various natural phenomena and for its application in various technological situations which are directly governed by the action of Coriolis force, magnetic force and viscous force. The subject of geophysical fluid dynamics nowadays has become an important branch of fluid dynamics due to the increasing interest to study environment. In geophysics, it is applied to measure and study the positions and velocities with respect to a fixed frame of reference on the surface of earth which rotate with respect to an inertial frame in the presence of its magnetic field. In astrophysics, it is applied to study the stellar and solar structure, inter planetary and inter stellar matter, solar storms and flares etc. The effect of the magnetic field on free convection flows is important in liquid metals, electrolytes and ionized gases. The effects of buoyancy forces on MHD forced convective flow have not received much attention. Ostrach [1] has studied the combined effects of a steady free and forced convective laminar flow and heat transfer between two vertical walls. The heat transfer aspect of MHD channel flows has been studied by Yen [2], Soundalgekar [3] and Jagadeesan [4]. Gupta [5] has studied the effects of buoyancy forces on a forced convective flow through a horizontal channel with nonconducting walls. Nanda and Mohanty [6] considered the hydromagnetic flow in a rotating channel formed by two infinite horizontal plates under the action of a constant pressure gradient. Assuminng the flow to be steady, fully devolved they have shown that when the applied magnetic field and rotation are weak, the effect of the magnetic field on the flow in the direction normal to the pressure gradient is unaffected by rotation. Also for a strong magnetic field they observed modified Ekman layers near the boundaries whose thickness is inversely proportional to the magnetic field and the shear stresses at the plates always decrease with increase in the magnetic parameter. Jana et al. [7] and Seth and Maiti [8] have presented detailed analysis of the flow of a viscous incompressible fluid through the rotating channel. Mohan [9] has studied the free convection effects for a similar configuration. It is found that when the Grashoff number is large, the fluid in the vicinity of the two plates move in the opposite directions and the flow separation take place only at the lower plate. Hall effects on unsteady MHD free and forced convection flow in a porous rotating channel have been investigated by Sivaprasad et al. [10]. The forced convective heat transfer in a MHD channel with Hall and Ion-slip currents has been presented by Mittal and Bhat [11]. Rao et al. [12] have studied the combined effects of free and forced convection on MHD flow in a rotating porous channel. The magnetohydrodynamic combined convective flow through a horizontal channel is studied by Mori [13], Yu [14], Datta and Jana [15], Ghosh and Bhattacharyya [16] and Pop et al. [17]. Guria et al. [18] have studied the Hall effects on the hydromagnetic convective flow through a rotating channel under general wall conditions. The effects of wall conductance on MHD fully developed flow with asymmetric heating of the wall have been investigated by Guria et al [19]. Seth et al. [20] have studied the combined free and forced convection MHD flow in a rotating channel with perfectly conducting walls. Analytical solution to the problem of MHD free convective flow of an electrically conducting fluid between two heated parallel plates in the presence of an induced magnetic field has been presented by Singha[21]. The combined free and forced convection flow of a viscous incompressible electrically conducting fluid in a rotating channel have been investigated by Seth et al [22]. Ahmed [23] has analyzed the mixed convection hydromagnetic oscillatory flow and periodic heat transfer of a viscous incompressible and electrically conducting fluid past an infinite vertical porous plate. The exact solution of MHD mixed convection periodic flow in a rotating vertical channel with heat radiation has been presented by Singh [24]. Seth et al. [25] have studied the combined free and forced convection CouetteHartmann flow in a rotating system with Hall effects.

In the present paper, we have studied the MHD free and forced convective flow of a viscous incompressible electrically conducting fluid in a rotating channel in the presence of a uniform transverse magnetic field on taking induced magnetic field into account. The governing equations are solved analytically. The upper plate is moving with a uniform velocity $U$ and the lower plate is kept fixed. The results obtained are discussed graphically. It is found that both the primary velocity $u_{1}$ and the absolute value of the secondary velocity $v_{1}$ decrease with an increase in either magnetic parameter $M^{2}$ or Grashof number $G r$. The induced magnetic field component $h_{x}$ and the absolute value of the induced magnetic field component $h_{y}$ decrease with an increase in either magnetic parameter $M^{2}$ or Grashof number $\mathrm{Gr}$. On the other hand, the induced magnetic field component $h_{x}$ decreases and the absolute value of the 
induced magnetic field component $h_{y}$ increases with an increase in rotation parameter $K^{2}$. It is also observed that the shear stress $\tau_{x_{1}}$ due to the primary flow increases and the shear stress $\tau_{y_{1}}$ due to the secondary flow at the upper plate $(\eta=1)$ decreases with an increase in magnetic parameter $M^{2}$. Further, the rate of heat transfer at the lower plate increases whereas the rate of heat transfer at the upper plate decreases with an increase in either magnetic parameter $M^{2}$ or Eckert number $E c$ or Grashof number $\mathrm{Gr}$.

\section{FORMULATION OF THE PROBLEM AND ITS SOLUTIONS}

Consider the fully-developed combined free and forced convective flow of an electrically conducting fluid bounded by a perfectly conducting plate at $z=0$ and a non-conducting plate at $z=d$ in a rotating system. Choose a Cartesian coordinates system such as $x$-axis is along the lower stationary plate in the direction of the flow, the $z$-axis perpendicular to the plates and the $y$-axis normal to $x z$-plane (see Fig.1). The upper plate is moving with a uniform velocity $U$ while the lower plate is held at rest. The plates and the fluid rotate in unison with a uniform angular velocity $\Omega$ about $z$-axis. A uniform magnetic field $H_{0}$ is applied perpendicular to the plates. The forced flow in the rotating channel is generated due to a constant pressure gradient. Since the plates are infinitely long, all physical variables, except pressure, depend on $z$ only. The equation of continuity $\nabla \cdot \vec{q}=0$ with no-slip condition at the plates gives $w=0$ everywhere in the flow where $\vec{q} \equiv(u, v, w)$. The solenoidal relation $\nabla \cdot \vec{H}=0$ gives $H_{z}=$ constant $=H_{0} \quad$ everywhere in the flow where $\vec{H} \equiv\left(H_{x}, H_{y}, H_{0}\right)$.

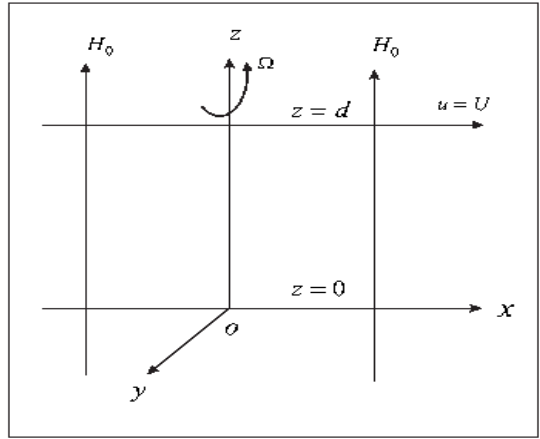

\section{Figure1: Geometry of the Problem}

The momentum equations for the fully developed steady flow in a rotating frame of reference are

$$
\begin{aligned}
& -2 \Omega \rho v=-\frac{\partial p}{\partial x}+\mu \frac{d^{2} u}{d z^{2}}+\mu_{e} H_{0} \frac{d H_{x}}{d z}, \\
& 2 \Omega \rho u=\mu \frac{d^{2} v}{d z^{2}}+\mu_{e} H_{0} \frac{d H_{y}}{d z}, \\
& 0=-\frac{\partial p}{\partial z}-\rho g-\frac{1}{2} \mu_{e}\left(\frac{d H_{x}^{2}}{d z}+\frac{d H_{y}^{2}}{d z}\right),
\end{aligned}
$$

where $\mu, \mu_{e}, \rho$ and $p$ are coefficient of viscosity, magnetic permeability, fluid density and modified fluid pressure including centrifugal force respectively.
The $x$ - and $y$ - components of the magnetic induction equations are

$$
\begin{aligned}
& v_{m} \frac{d^{2} H_{x}}{d z^{2}}+H_{0} \frac{d u}{d z}=0, \\
& v_{m} \frac{d^{2} H_{y}}{d z^{2}}+H_{0} \frac{d v}{d z}=0,
\end{aligned}
$$

where $v_{m}=\frac{1}{\mu_{e} \sigma}$ and $\sigma$ is the electrical conductivity of the fluid.

The boundary conditions for the velocity and the induced magnetic fields are

$$
\begin{aligned}
& u=0, v=0, \frac{d H_{x}}{d z}=\frac{d H_{y}}{d z}=0 \text { at } z=0, \\
& u=U, v=0, H_{x}=0, H_{y}=0 \text { at } z=d .
\end{aligned}
$$

Assuming uniform axial temperature variation along the plates, the temperature of the fluid can be written as

$$
T-T_{0}=N x+\phi(z),
$$

where $N$ is the uniform temperature gradient along $x$ direction, $\phi(z)$ an arbitrary function of $z, T$ the fluid temperature and $T_{0}$ the temperature in the reference state.

The equation of state under the Boussinesq approximation is assumed to be

$$
\rho=\rho_{0}\left[1-\beta^{*}\left(T-T_{0}\right)\right],
$$

where $\beta^{*}$ is the coefficient of thermal expansion and $\rho_{0}$ the fluid density in the reference state.

On the use of (8), the equation (3) becomes

$$
0=-\frac{\partial p}{\partial z}-\rho_{0} g\left[1-\beta^{*}\left(T-T_{0}\right)\right]-\frac{1}{2} \mu_{e}\left(\frac{d H_{x}^{2}}{d z}+\frac{d H_{y}^{2}}{d z}\right)
$$

Integrating the equation (9) with respect to $z$, we get

$$
p=-\rho_{0} g \int\left[1-\beta^{*}\left(T-T_{0}\right)\right] d z-\frac{1}{2} \mu_{e}\left(H_{x}^{2}+H_{y}^{2}\right)+F(x),(10)
$$

where $F(x)$ is an unknown function.

On the use of (7) and (10), equations (1) and (2) become

$$
\begin{aligned}
& -2 \Omega v=-\frac{1}{\rho_{0}} \frac{\partial F}{\partial x}-g \beta^{*} N z+v \frac{d^{2} u}{d z^{2}}+\frac{\mu_{e} H_{0}}{\rho} \frac{d H_{x}}{d z}, \\
& 2 \Omega u=v \frac{d^{2} v}{d z^{2}}+\frac{\mu_{e} H_{0}}{\rho} \frac{d H_{y}}{d z} .
\end{aligned}
$$

Introducing the non-dimensional variables

$$
\eta=\frac{z}{d},\left(u_{1}, v_{1}\right)=\frac{(u, v)}{U P_{x}},\left(h_{x}, h_{y}\right)=\frac{\left(H_{x}, H_{y}\right)}{\sigma \mu_{e} U d H_{0} P_{x}},
$$

equations (11), (12), (4) and (5) become

$$
\begin{aligned}
& -2 K^{2} v_{1}=1-G r \eta+\frac{d^{2} u_{1}}{d \eta^{2}}+M^{2} \frac{d h_{x}}{d \eta}, \\
& 2 K^{2} u_{1}=\frac{d^{2} v_{1}}{d \eta^{2}}+M^{2} \frac{d h_{y}}{d \eta}, \\
& 0=\frac{d^{2} h_{x}}{d \eta^{2}}+\frac{d u_{1}}{d \eta}, \\
& 0=\frac{d^{2} h_{y}}{d \eta^{2}}+\frac{d v_{1}}{d \eta},
\end{aligned}
$$

where $M^{2}=\frac{\sigma \mu_{e}^{2} H_{0}^{2} d^{2}}{\rho_{0} v}$ is the magnetic parameter which is square of Hartmann number, $K^{2}=\frac{\Omega d^{2}}{v}$ the rotation parameter 
which is reciprocal of Ekman number, $G r=\frac{g \beta^{*} N d^{3}}{v U P_{x}}$ the Grashof number and $P_{x}=-\frac{d^{2}}{\rho_{0} v U} \frac{\partial F}{\partial x}$ the non-dimensional fluid pressure gradient.

Equation (7) shows that positive or negative value of $N$ corresponds to heating or cooling of the channel plates along $x$-direction. Then from the expression of $G r$ it is followed that $G r>$ or $<0$ according as the channel plates are heated or cooled in the axial direction.

Combining equations (14) and (15) and equations (16) and (17), we get

$$
\begin{aligned}
& \frac{d^{2} f}{d \eta^{2}}+M^{2} \frac{d h}{d \eta}-2 i K^{2} f=-1+G r \eta \\
& \frac{d^{2} h}{d \eta^{2}}+\frac{d f}{d \eta}=0,
\end{aligned}
$$

where

$$
f=u_{1}+i v_{1}, h=h_{x}+i h_{y} \text { and } i=\sqrt{-1} .
$$

The corresponding boundary conditions for $f(\eta)$ and $h(\eta)$ are

$$
\begin{aligned}
& f=0 \text { at } \eta=0 \text { and } f=1 \text { at } \eta=1, \\
& \frac{d h}{d \eta}=0 \text { at } \eta=0 \text { and } h=0 \text { at } \eta=1 .
\end{aligned}
$$

Solutions of the equations (18) and (19) subject to the boundary conditions (21) are

$$
\begin{aligned}
f(\eta) & =\frac{1}{\lambda^{2}}\left[1-\frac{\sinh \lambda \eta}{\sinh \lambda}-\frac{\sinh \lambda(1-\eta)}{\sinh \lambda}\right] \\
& +\frac{G r}{\lambda^{2}}\left[\frac{\sinh \lambda \eta}{\sinh \lambda}-\eta\right]+\frac{\sinh \lambda \eta}{\sinh \lambda} \\
h(\eta) & =\frac{\cosh \lambda-\cosh \lambda \eta}{\lambda \sinh \lambda} \\
& +\frac{1}{\lambda^{2}}\left[1-\eta-\frac{\cosh \lambda-\cosh \lambda \eta}{\lambda \sinh \lambda}+\frac{1-\cosh \lambda(1-\eta)}{\lambda \sinh \lambda}\right] \\
& +\frac{G r}{\lambda^{2}}\left[\frac{\cosh \lambda-\cosh \lambda \eta}{\lambda \sinh \lambda}-\frac{1}{2}\left(1-\eta^{2}\right)\right]
\end{aligned}
$$

where

$$
\lambda=\alpha+i \beta \text { and } \alpha, \beta= \pm \frac{1}{\sqrt{2}}\left[\left(M^{4}+4 K^{4}\right)^{\frac{1}{2}} \pm M^{2}\right]^{\frac{1}{2}} \text {. }
$$

On separating into a real and imaginary parts one can easily obtain the velocity components $u_{1}$ and $v_{1}$ from the equation (22) and the induced magnetic field components $h_{x}$ and $h_{y}$ from the equation (23). In the absence of Hall currents $(m=0)$, equations (22) and (23) are not exactly identical with equations (22) and (23) of Seth et al.[25] due to the different boundary conditions.

\section{RESULTS AND DISCUSSION}

We have presented the non-dimensional primary velocity $u_{1}$ and secondary velocity $v_{1}$ against $\eta$ for several values of magnetic parameter $M^{2}$, rotation parameter $K^{2}$ and Grashof number $G r$ in Figs.2-4. Figs.2 and 3 show that the primary velocity $u_{1}$ and the absolute value of the secondary velocity $v_{1}$ decrease with an increase in either magnetic parameter $M^{2}$ or Grashof number $G r$. It is seen from Fig.4 that the primary velocity $u_{1}$ decreases whereas the absolute value of the secondary velocity $v_{1}$ decreases in the vicinity of the lower plate and increases near the upper plate with an increase in rotation parameter $K^{2}$. The rotation parameter $K^{2}=\frac{\Omega d^{2}}{v}$ defines the relative magnitude of the Coriolis force and the viscous force, therefore it is clear that high magnitude Coriolis forces are counter-productive for the primary flow. Also, we have presented the non-dimensional primary induced magnetic field component $h_{x}$ and the secondary induced magnetic field component $h_{y}$ against $\eta$ for several values of magnetic parameter $M^{2}$, rotation parameter $K^{2}$ and Grashof number $G r$ in Figs.5-7. It is revealed from Figs.5 and 6 that both the induced magnetic field component $h_{x}$ and the absolute value of the secondary induced magnetic field component $h_{y}$ decrease with an increase in either magnetic parameter $M^{2}$ or Grashof number $G r$. It means that both the magnetic field and buoyancy force have a tendency to reduce the primary and the secondary induced magnetic field components. Fig. 7 shows that an increase in rotation parameter $K^{2}$ leads to reduce the primary induced magnetic field component $h_{x}$ and accelerates the absolute value of the secondary induced magnetic field component $h_{y}$.

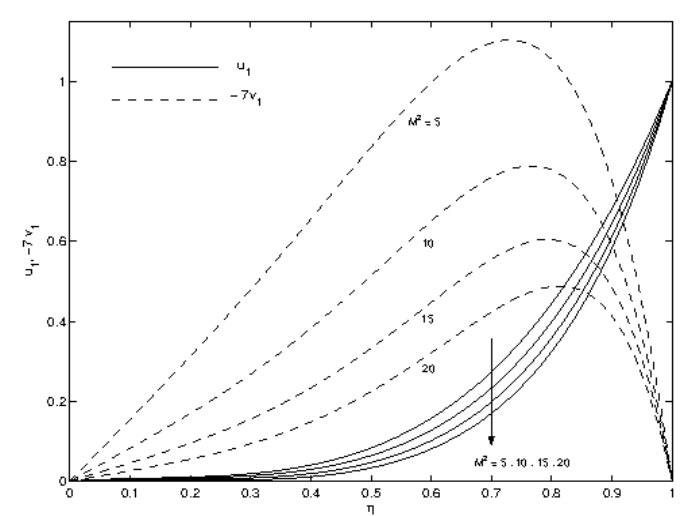

Figure 2: Primary and secondary velocities for different $M^{2}$ when $G r=5$ and $K^{2}=5$

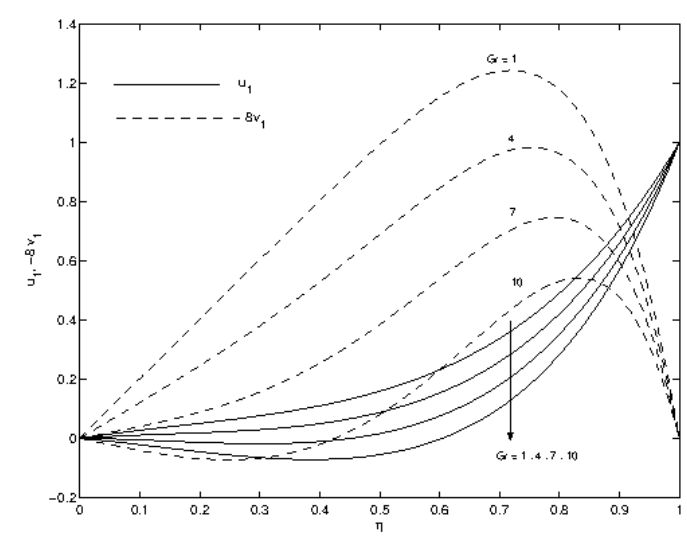

Figure 3: Primary and secondary velocities for different $G r$ when $M^{2}=10$ and $K^{2}=5$ 


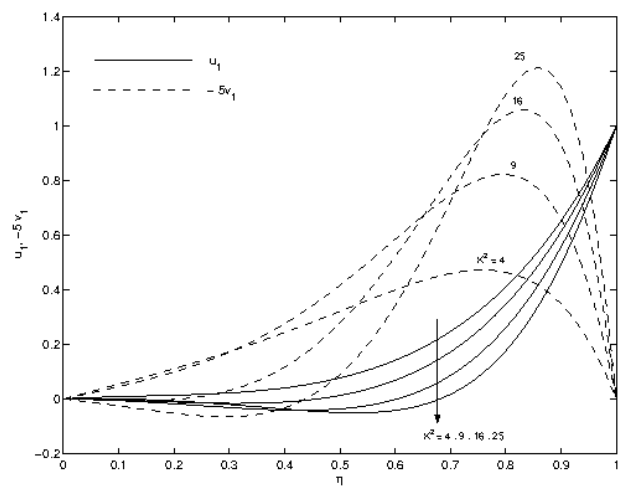

Figure 4: Primary and secondary velocities for different $K^{2}$ when $M^{2}=10$ and $G r=5$

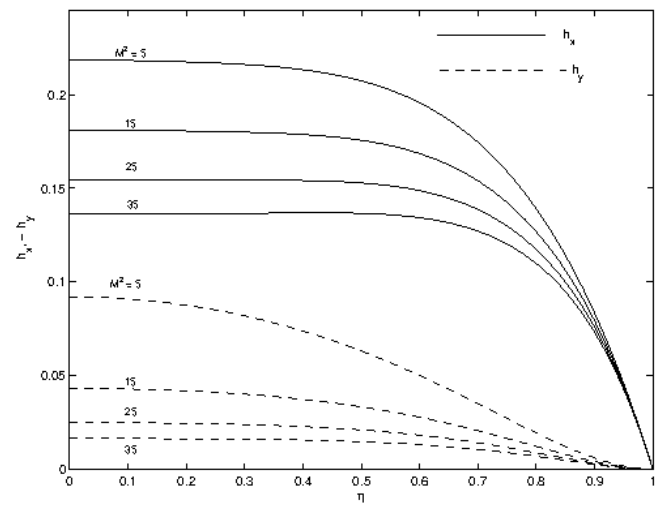

Figure 5: The induced magnetic field components for different $M^{2}$ when $G r=5$ and $K^{2}=5$

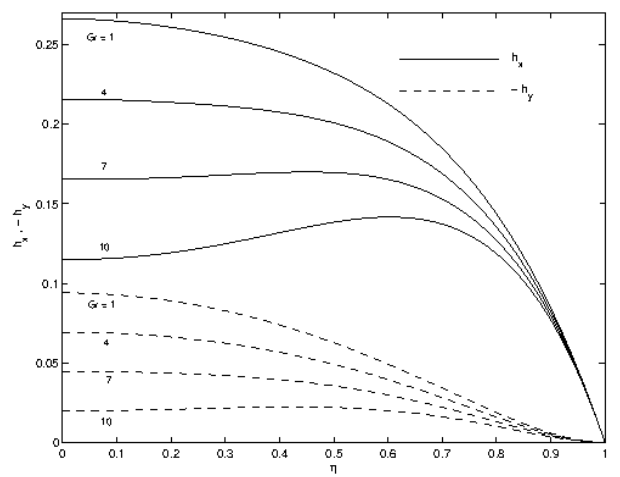

Figure 6: The induced magnetic field components for different $G r$ when $M^{2}=10$ and $K^{2}=5$

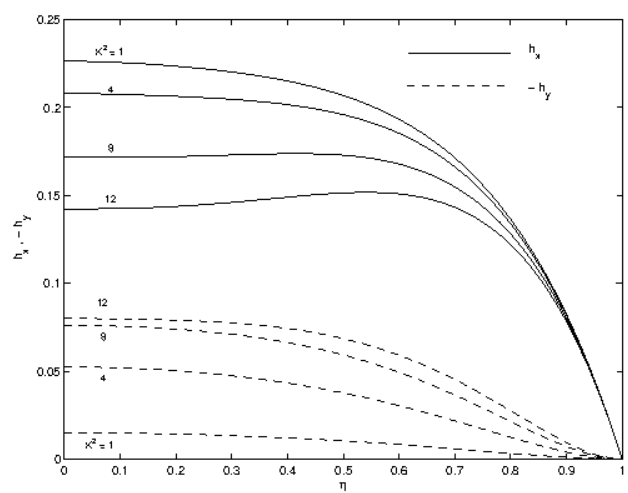

Figure 7: The induced magnetic field components for different $K^{2}$ when $M^{2}=10$ and $G r=5$
The non-dimensional shear stress components due to the primary and secondary flows at the lower plate $\eta=0$ are respectively

$$
\begin{gathered}
\tau_{x_{0}}=\frac{2}{\left(\alpha^{2}+\beta^{2}\right)(\cosh 2 \alpha-\cos 2 \beta)}\left[-a_{1}+\frac{a_{2} G r}{\alpha^{2}+\beta^{2}}+a_{3}\right], \\
\tau_{y_{0}}=\frac{2}{\left(\alpha^{2}+\beta^{2}\right)(\cosh 2 \alpha-\cos 2 \beta)}\left[b_{1}-\frac{b_{2} G r}{\alpha^{2}+\beta^{2}}+b_{3}\right] .
\end{gathered}
$$

Similarly, the non-dimensional shear stress components due to the primary and secondary flows at the upper plate $\eta=1$ are respectively

$\tau_{x_{1}}=\frac{2}{\left(\alpha^{2}+\beta^{2}\right)(\cosh 2 \alpha-\cos 2 \beta)}\left[a_{1}+\frac{a_{2}^{*} G r}{\alpha^{2}+\beta^{2}}+a_{3}^{*}\right]$,

$\tau_{y_{1}}=\frac{2}{\left(\alpha^{2}+\beta^{2}\right)(\cosh 2 \alpha-\cos 2 \beta)}\left[-b_{1}-\frac{b_{2}^{*} G r}{\alpha^{2}+\beta^{2}}+b_{3}^{*}\right]$, where

$a_{1}=\alpha \sinh \alpha \cos \beta-\beta \cosh \alpha \sin \beta-\frac{1}{2}(\alpha \sinh 2 \alpha-\beta \sin 2 \beta)$,

$b_{1}=\alpha \cosh \alpha \sin \beta+\beta \sinh \alpha \cos \beta-\frac{1}{2}(\alpha \sin 2 \beta+\beta \sinh 2 \alpha)$,

$a_{2}=\left(\alpha^{2}+\beta^{2}\right)(\alpha \sinh \alpha \cos \beta-\beta \cosh \alpha \sin \beta)$

$-\frac{1}{2}\left(\alpha^{2}-\beta^{2}\right)(\cosh 2 \alpha-\cos 2 \beta)$,

$b_{2}=\left(\alpha^{2}+\beta^{2}\right)(\alpha \cosh \alpha \sin \beta+\beta \sinh \alpha \cos \beta)$

$-\alpha \beta(\cosh 2 \alpha-\cos 2 \beta)$,

$a_{3}=\left(\alpha^{2}+\beta^{2}\right)(\alpha \sinh \alpha \cos \beta+\beta \cosh \alpha \sin \beta)$,

$b_{3}=\left(\alpha^{2}+\beta^{2}\right)(\beta \sinh \alpha \cos \beta-\alpha \cosh \alpha \sin \beta)$,

$a_{2}^{*}=\frac{1}{2}\left[\left(\alpha^{2}+\beta^{2}\right)(\alpha \sinh 2 \alpha-\beta \sin 2 \beta)\right.$

$\left.-\left(\alpha^{2}-\beta^{2}\right)(\cosh 2 \alpha-\cos 2 \beta)\right]$,

$b_{2}^{*}=\frac{1}{2}\left[\left(\alpha^{2}+\beta^{2}\right)(\alpha \sin 2 \beta+\beta \sinh 2 \alpha)\right.$

$-2 \alpha \beta(\cosh 2 \alpha-\cos 2 \beta)]$,

$a_{3}^{*}=\frac{1}{2}\left(\alpha^{2}+\beta^{2}\right)(\alpha \sinh 2 \alpha+\beta \sin 2 \beta)$,

$b_{3}^{*}=\frac{1}{2}\left(\alpha^{2}+\beta^{2}\right)(\beta \sinh 2 \alpha-\alpha \sin 2 \beta)$.

It is interesting to note that the shear stress components due to the primary and secondary flows do not vanish at the plates $\eta=0$ and $\eta=1$ and they depend on both magnetic parameter $M^{2}$ and rotation parameter $K^{2}$ when $G r=0$. Thus, we deduce an interesting conclusion that for conducting plates there are no flow reversal in the absence of buoyancy force $G r=0$.

Numerical results of the shear stresses at the plates $\eta=0$ and $\eta=1$ are depicted in Figs.8-11 against magnetic parameter $M^{2}$ for several values of Grashof number $\mathrm{Gr}$ and rotation parameter $K^{2}$. Fig.8 shows that both the shear stress $\tau_{x_{0}}$ due to the primary flow and the absolute value of the shear stress $\tau_{y_{0}}$ due to the secondary flow at the lower plate $\eta=0$ decrease with an increase in Grashof number $G r$. This implies that the buoyancy force has a tendency to reduce the shear stresses at 
the lower plate. It is seen from Fig.9 that the shear stress $\tau_{x_{0}}$ decreases and the absolute value of the shear stress $\tau_{y_{0}}$ increases with an increase in rotation parameter $K^{2}$. This means that rotation has tendency to reduce the primary shear stress and to enhance the secondary shear stress at the lower plate $(\eta=0)$. Further, it is illustrated from Fig.10 that the shear stress $\tau_{x_{1}}$ due to the primary flow increases while the shear stress $\tau_{y_{1}}$ due to the secondary flow at the upper plate $(\eta=1)$ decreases with an increase in Grashof number $G r$. It implies that the buoyancy force has a tendency to enhance the primary shear stress and to reduce the secondary shear stress at the upper plate. It is seen from Fig.11 that the shear stress $\tau_{x_{1}}$ and the shear stress $\tau_{y_{1}}$ increase with an increase in rotation parameter $K^{2}$. Thus, the rotation has a tendency to enhance the shear stresses at the upper plate. Further, it is found from Fig. 8 that the the shear stress $\tau_{x_{0}}$ increases whereas the shear stress $\tau_{y_{0}}$ decreases with an increase in magnetic parameter $M^{2}$ for fixed value of $G r$. Fig.9 reveals that both the shear stresses $\tau_{x_{0}}$ and $\tau_{y_{0}}$ decrease with an increase in magnetic parameter $M^{2}$ for fixed value of $K^{2}$. Figs. 10 and 11 show that the shear stress $\tau_{x_{1}}$ increases and the shear stress $\tau_{y_{1}}$ decreases with an increase in magnetic parameter $M^{2}$ for fixed values of $G r$ and $K^{2}$. Thus, magnetic field has a tendency to enhance the primary and to reduce the secondary shear stress at the upper plate.

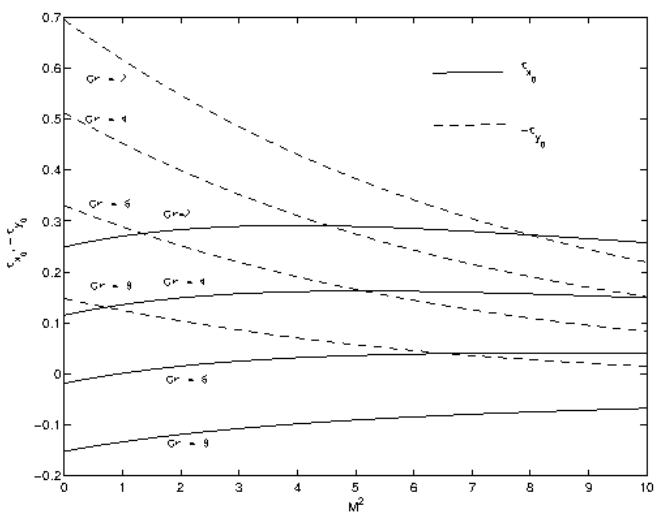

Figure 8: Shear stresses $\tau_{x_{0}}$ and $\tau_{y_{0}}$ for different $G r$ when $K^{2}=5$

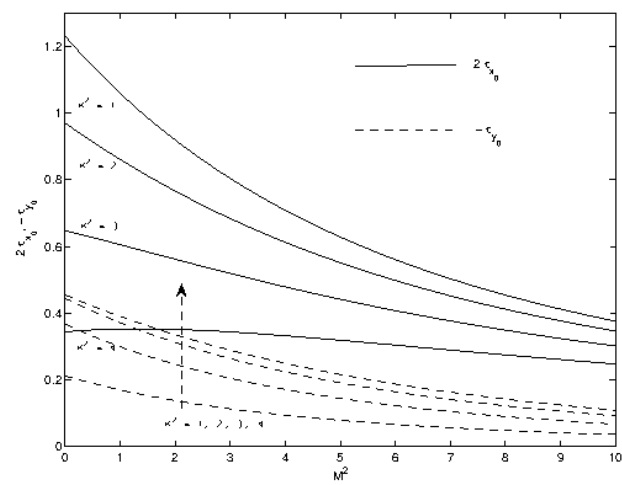

Figure 9: Shear stresses $\tau_{x_{0}}$ and $\tau_{y_{0}}$ for different $K^{2}$ when $G r=5$

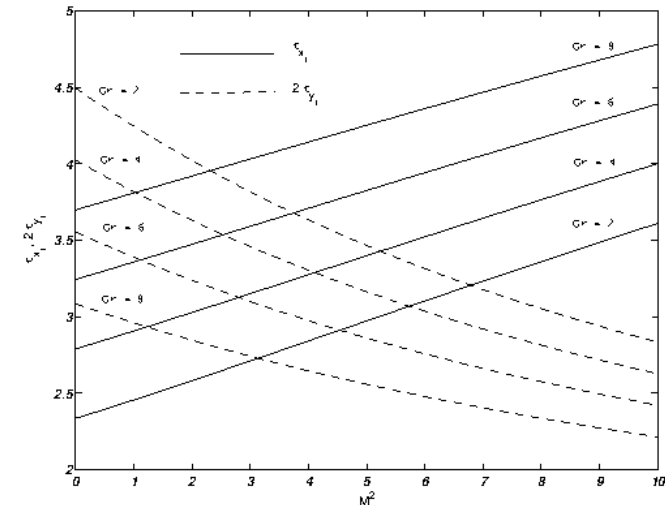

Figure 10: Shear stresses $\tau_{x_{1}}$ and $\tau_{y_{1}}$ for different $G r$ when $K^{2}=5$

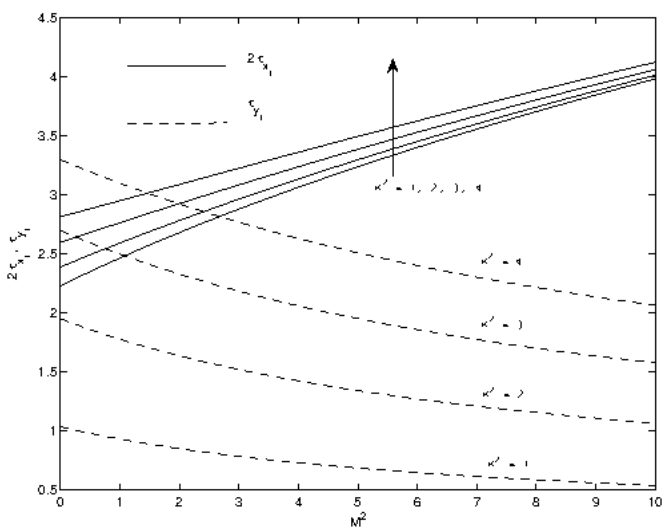

Figure 11: Shear stresses $\tau_{x_{1}}$ and $\tau_{y_{1}}$ for different $K^{2}$ when

$$
G r=5
$$

The shear stresses at the plate $\eta=0$ due to the primary and secondary flows respectively will vanish if the critical Grashof numbers are as

$$
\begin{aligned}
& G r_{x_{0}}=\frac{a_{1}-a_{3}}{a_{2}}\left(\alpha^{2}+\beta^{2}\right), \\
& G r_{y_{0}}=\frac{b_{1}+b_{3}}{b_{2}}\left(\alpha^{2}+\beta^{2}\right),
\end{aligned}
$$

where $\alpha$ and $\beta$ are given by (24), $a_{1}, a_{2}, a_{3}, b_{1}, b_{2}$ and $b_{3}$ are given by (29).

Similarly, the primary and secondary shear stresses will vanish at the upper plate $\eta=1$ if the critical Grashof numbers are as

$$
\begin{aligned}
& G r_{x_{1}}=-\frac{a_{1}+a_{3}^{*}}{a_{2}^{*}}\left(\alpha^{2}+\beta^{2}\right), \\
& G r_{y_{1}}=-\frac{b_{1}-b_{3}^{*}}{b_{2}^{*}}\left(\alpha^{2}+\beta^{2}\right),
\end{aligned}
$$

where $\alpha$ and $\beta$ are given by (24), $a_{1}, a_{2}^{*}, a_{3}^{*}, b_{1}, b_{2}^{*}$ and $b_{3}^{*}$ are given by (29). We have computed the critical Grashof numbers for which the primary and secondary flows reversal occur at the lower and upper plate for several values of magnetic parameter $M^{2}$ and rotation parameter $K^{2}$ and are presented in Tables 1 and 2. It is seen from Table 1 that the critical Grashof number at the lower plate for the primary flow as well as for the secondary flow reversal decreases with an increase in rotation parameter $K^{2}$ for fixed value of magnetic parameter $M^{2}$. Further, the 
critical Grashof number at the lower plate for the primary flow reversal increases whereas for the secondary flow reversal decreases with an increase in magnetic parameter $M^{2}$ for fixed value of rotation parameter $K^{2}$. It is observed from Table 2 that the absolute value of critical Grashof number for the primary flow as well as critical Grashof number for the secondary flow at the upper plate increase with an increase in either rotation parameter $K^{2}$ or magnetic parameter $M^{2}$.

Table 1. Critical Grashof numbers at the lower plate

\begin{tabular}{|c|c|c|c|c|c|c|c|c|}
\hline & \multicolumn{4}{|c|}{$G r_{x_{0}}$} & \multicolumn{4}{c|}{$G r_{y_{0}}$} \\
\hline$M^{2} \backslash K^{2}$ & 6 & 8 & 10 & 12 & 6 & 8 & 10 & 12 \\
& & & & & & & & \\
\hline 10 & 6.28842 & 5.29808 & 4.25558 & 3.30211 & 8.31294 & 7.73973 & 7.06652 & 6.34383 \\
15 & 6.60528 & 6.07865 & 5.56441 & 5.15921 & 7.68155 & 7.23320 & 6.70448 & 6.13039 \\
20 & 6.77021 & 6.48801 & 6.23118 & 6.06387 & 7.16846 & 6.82050 & 6.40910 & 5.95907 \\
25 & 6.91035 & 6.77003 & 6.65852 & 6.61841 & 6.73376 & 6.46536 & 6.14712 & 5.79709 \\
\hline
\end{tabular}

Table 2. Critical Grashof numbers at the upper plate

\begin{tabular}{|c|c|c|c|c|c|c|c|c|}
\hline & \multicolumn{4}{|c|}{$-10^{-2} G r_{x_{1}}$} & \multicolumn{4}{c|}{$10^{-2} G r_{y_{1}}$} \\
\hline$M^{2} \backslash K^{2}$ & 6 & 8 & 10 & 12 & 6 & 8 & 10 & 12 \\
\hline 10 & 0.17779 & 0.20 .714 & 0.23997 & 0.27489 & 0.30659 & 0.33437 & 0.36684 & 0.40262 \\
15 & 0.22445 & 0.24 .872 & 0.27691 & 0.30790 & 0.35330 & 0.37705 & 0.40539 & 0.43726 \\
20 & 0.27369 & 0.29415 & 0.31856 & 0.34604 & 0.40334 & 0.42387 & 0.44874 & 0.47717 \\
25 & 0.32427 & 0.34186 & 0.36321 & 0.38768 & 0.45523 & 0.47318 & 0.49518 & 0.52065 \\
\hline
\end{tabular}

\section{HEAT TRANSFER}

The energy equation for the fully developed flow including the viscous and Joule dissipations is

$k \frac{d^{2} T}{d z^{2}}+\mu\left[\left(\frac{d u}{d z}\right)^{2}+\left(\frac{d v}{d z}\right)^{2}\right]+\frac{1}{\sigma}\left[\left(\frac{d H_{x}}{d z}\right)^{2}+\left(\frac{d H_{y}}{d z}\right)^{2}\right]=0$,

where $k$ is the thermal conductivity and $\mu$ the dynamic viscosity.

Using (7) and (14), the equation (34) can be written in a nondimensional form as

$$
\frac{d^{2} \theta}{d \eta^{2}}+\operatorname{Pr} E c\left[\left(\frac{d u_{1}}{d \eta}\right)^{2}+\left(\frac{d v_{1}}{d \eta}\right)^{2}+M^{2}\left\{\left(\frac{d h_{x}}{d \eta}\right)^{2}+\left(\frac{d h_{y}}{d \eta}\right)^{2}\right\}\right]=0
$$

where $\operatorname{Pr}=\frac{\mu C_{p}}{k}$ is the Prandtl number, $E c=\frac{U^{2}}{C_{p}\left(T_{1}-T_{0}\right)}$ the Eckert number, $\theta(\eta)=\frac{\phi(z)}{N L P}, C_{p}$ the specific heat at constant pressure and $T_{1}$ denote the uniform temperature of the plate at $z=d$.

For the temperature boundary conditions, we assume the reference temperature $T_{0}$ in such a way that the temperature at the lower plate $(\eta=0)$ is $T_{0}+N x$ and then by virtue of the equation (7), we have $\phi(-1)=0$. Hence using (7) the boundary conditions for $\theta(\eta)$ are given by

$$
\theta(0)=0 \text { and } \theta(1)=\frac{\phi(1)}{N L}=A \text { (say), }
$$

where $A$ is taken as the plate temperature parameter.

Substituting the values of $u_{1}, v_{1}, h_{x}$ and $h_{y}$ from equations (22) and (23) into (35) and solving the resulting differential equation subject to the boundary condition (36), one can obtain temperature distribution $\theta(\eta)$. We avoid the expression of fluid temperature $\theta(\eta)$ as it is lengthy.
Effects of magnetic parameter $M^{2}$, rotation parameter $K^{2}$, Grashof number $G r$ and Eckert number $E c$ on the fluid temperature distribution have been shown in Figs.12-15 when $\operatorname{Pr}=3$ and $A=1$. It is seen from Fig.12 that the fluid temperature $\theta(\eta)$ decreases with an increase in rotation parameter $K^{2}$. This implies that rotation tends to reduce fluid temperature. Figs.13-15 show that the fluid temperature $\theta(\eta)$ increases with an increase in either magnetic parameter $M^{2}$ or Grashof number $G r$ or Eckert number $E c$. This implies that magnetic field and Grashof number tend to enhance fluid temperature. An increase in Eckert number lead to increase the fluid temperature. This is due to the heat energy stored in the fluid because of the frictional heating.

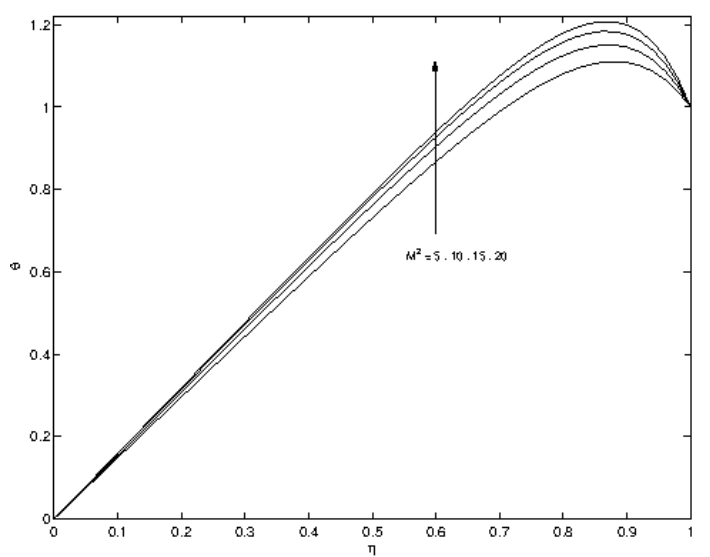

Figure 12: Temperature $\theta$ for different $M^{2}$ when $G r=5$, $K^{2}=10$ and $E c=0.1$ 


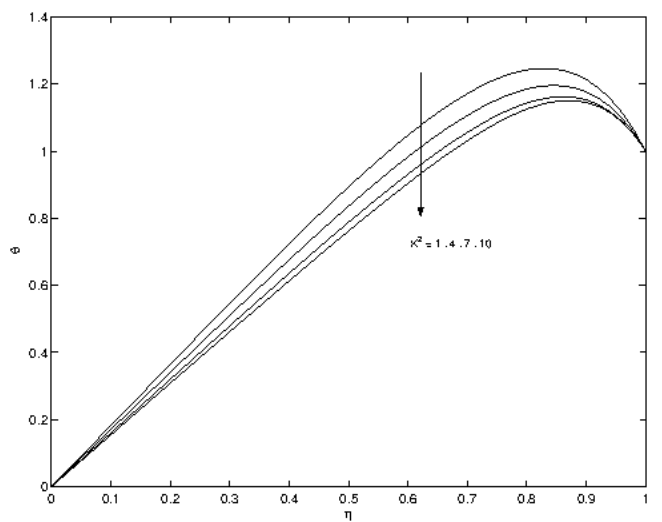

Figure 13: Temperature $\theta$ for different $K^{2}$ when $G r=5$, $M^{2}=10$ and $E c=0.1$

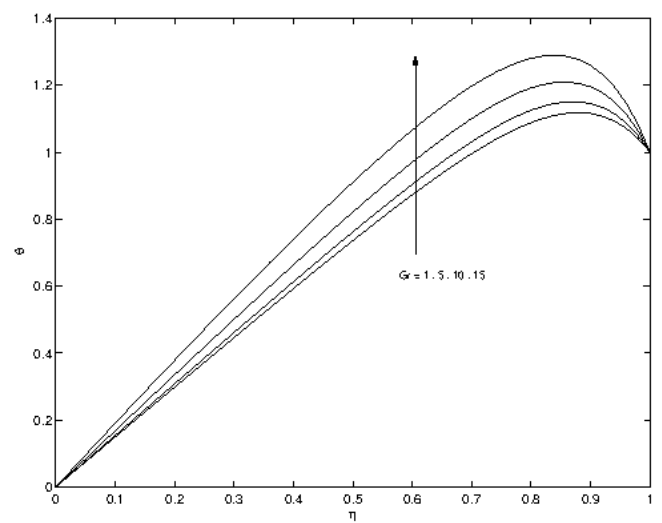

Figure 14: Temperature $\theta$ for different $G r$ when $M^{2}=10$, $K^{2}=10$ and $E c=0.1$

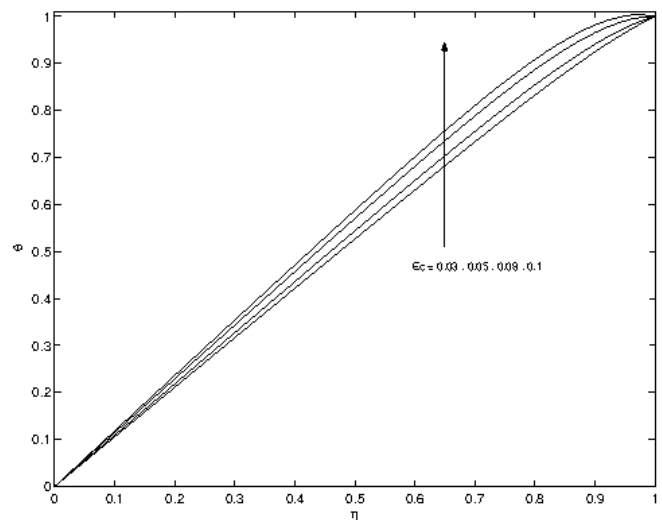

Figure 15: Temperature $\theta$ for different $E c$ when $M^{2}=10$, $K^{2}=10$ and $G r=5$

The numerical values of the rate of heat transfer $\left(\frac{d \theta}{d \eta}\right)_{\eta=0}$ at the lower plate and $\left(\frac{d \theta}{d \eta}\right)_{\eta=1}$ at the upper plate are entered in

Tables 3-5 for several values of magnetic parameter $M^{2}$, rotation parameter $K^{2}$, Eckert number $E c$ and Grashof number $G r$ when $P r=0.03$ and $A=1$. It is seen from Tables 3 and 5 that the rate of heat transfer at the lower plate increases whereas it decreases at the upper plate with an increase in either magnetic parameter $M^{2}$ or Eckert number $E c$ for fixed value of Grashof number $\mathrm{Gr}$. It is observed from Table 4 that the rate of heat transfer at the lower plate $(\eta=0)$ decreases while it increases at the upper plate $(\eta=1)$ with an increase in rotation parameter $K^{2}$ for fixed value of Grashof number $G r$. Further, it is seen from Tables 3-5 that the rate of heat transfer at the lower plate increases whereas it decreases at the upper plate with an increase in Grashof number $\mathrm{Gr}$.

Table 3. Rate of heat transfers $\left(\frac{d \theta}{d \eta}\right)_{\eta=0}$ and $\left(\frac{d \theta}{d \eta}\right)_{\eta=1}$ when $K^{2}=10$ and $E c=0.1$

\begin{tabular}{|c|c|c|c|c|c|c|c|c|}
\hline & \multicolumn{4}{|c|}{$\left(\frac{d \theta}{d \eta}\right)_{\eta=0}$} & \multicolumn{4}{|c|}{$\left(\frac{d \theta}{d \eta}\right)_{\eta=1}$} \\
\hline$G r \backslash M^{2}$ & 5 & 10 & 15 & 20 & 5 & 10 & 15 & 20 \\
\hline 2 & 1.00159 & 1.00170 & 1.00175 & 1.00177 & 0.99027 & 0.98850 & 0.98679 & 0.98522 \\
\hline 4 & 1.00160 & 1.00177 & 1.00187 & 1.00191 & 0.98977 & 0.98767 & 0.98572 & 0.98400 \\
\hline 6 & 1.00165 & 1.00190 & 1.00203 & 1.00209 & 0.98917 & 0.98675 & 0.98455 & 0.98268 \\
\hline 8 & 1.00175 & 1.00207 & 1.00225 & 1.00232 & 0.98847 & 0.98572 & 0.98328 & 0.98127 \\
\hline
\end{tabular}

Table 4. Rate of heat transfers $\left(\frac{d \theta}{d \eta}\right)_{\eta=0}$ and $\left(\frac{d \theta}{d \eta}\right)_{\eta=1}$ when $M^{2}=10$ and $E c=0.1$

\begin{tabular}{|c|c|c|c|c|c|c|c|c|}
\hline & \multicolumn{4}{|c|}{$\left(\frac{d \theta}{d \eta}\right)_{\eta=0}$} & \multicolumn{4}{c|}{$\left(\frac{d \theta}{d \eta}\right)_{\eta=1}$} \\
\hline$G r \backslash K^{2}$ & 1 & 2 & 3 & 4 & 1 & 2 & 3 & 4 \\
\hline 2 & 1.00220 & 1.00215 & 1.00207 & 1.00199 & 0.98883 & 0.98897 & 0.98912 & 0.98922 \\
4 & 1.00258 & 1.00249 & 1.00236 & 1.00223 & 0.98628 & 0.98662 & 0.98703 & 0.98740 \\
6 & 1.00306 & 1.00292 & 1.00274 & 1.00255 & 0.98355 & 0.98409 & 0.98478 & 0.98542 \\
8 & 1.00363 & 1.00345 & 1.00321 & 1.00296 & 0.98064 & 0.98140 & 0.98235 & 0.98328 \\
\hline
\end{tabular}


Table 5. Rate of heat transfers $\left(\frac{d \theta}{d \eta}\right)_{\eta=0}$ and $\left(\frac{d \theta}{d \eta}\right)_{\eta=1}$ when $K^{2}=10$ and $M^{2}=10$

\begin{tabular}{|c|c|c|c|c|c|c|c|c|}
\hline & \multicolumn{5}{|c|}{$\left(\frac{d \theta}{d \eta}\right)_{\eta=0}$} & \multicolumn{4}{c|}{$\left(\frac{d \theta}{d \eta}\right)_{\eta=1}$} \\
\hline$G r \backslash E c$ & 0.05 & 0.06 & 0.08 & 0.1 & 0.05 & 0.06 & 0.08 & 0.1 \\
\hline 2 & 1.00085 & 1.00102 & 1.00136 & 1.00169 & 0.99425 & 0.99310 & 0.99080 & 0.98850 \\
4 & 1.00089 & 1.00106 & 1.00142 & 1.00177 & 0.99384 & 0.99260 & 0.99014 & 0.98767 \\
6 & 1.00095 & 1.00114 & 1.00152 & 1.00189 & 0.99337 & 0.99205 & 0.98940 & 0.98675 \\
8 & 1.00103 & 1.00124 & 1.00166 & 1.00206 & 0.99286 & 0.99143 & 0.98858 & 0.98572 \\
\hline
\end{tabular}

\section{CONCLUSION}

The forced flow in the rotating channel is generated deu to a constant pressure gradient and the interaction of the magnetic field with the effects of free convection on this forced field has been investigated. It is found that the magnetic field and the thermal buoyancy force have a retarding influence on the velocity as well as induced magnetic field components. The thermal buoyancy force tends to reduce the primary as well as secondary induced magnetic field components. It has less effect on the secondary induced magnetic field. The velocity and induced magnetic fields have been significantly affected by rotation. The rotation has same effect on the induced magnetic field components as thermal buoyancy force. The shear stresses at the plates depend on both the magnetic field as well as rotation even in the absence of buoyancy force. The fluid temperature increases with an increase in either magnetic parameter or Eckert number or Grashof number while it decreases on increasing of rotation parameter. Further, the rate of heat transfer at the lower plate increases whereas it decreases at the upper plate with an increase in Grashof number.

\section{REFERENCES}

[1] Ostrach, S.(1954). Combined natural and forced convection laminar flow and heat transfer of fluids with or without heat sources in channels with linearly varying wall temperature. Technical Report, NASA, USA. 31-41 .

[2] Yen, J. T. (1963). Efeect of wall electrical conductance on magnetohydrodynamics heat transfer in a channel. J. Heat Transfer. Tr. ASME. 85C: 371.

[3] Soundalgekar, V. M. (1969). On heat transfer in crossed fluids MHD channel flow between conducting walls. Proc. Nat. Inst. Sci. India. 35: 329.

[4] Jagdeesan, K.(1964). Heat transfer due to hydromagnetic channel flow with conducting walls. AIAA J. 2: 756.

[5] Gupta, A. S. (1969). Combined free and forced convection effects on the magnetohydrodynamic flow through a channel. Z. Angew. Math. Phys. 20: 506-513.

[6] Nanda, R.S. and Mohanty, H. K. (1970). Hydromagnetic flow in a rotating channel, Appl. Sci. Res. 24: 65-78.

[7] Jana, R. N., Datta, N. and Mazumder, B. S. (1977). Magnetohydrodynamic Couette flow and heat transfer in a rotating system. J. Phys. Soc. Jpn. 42: 1034-1039.

[8] Seth, G.S. and Maiti, M. K. (1982).MHD Couette flow and heat transfer in a rotating system. Indian J. Pure Appl. Math. 13: 931-945.
[9] Mohan, M. (1977): Combined effects of free and forced convection on magnetohydrodynamic flow in a rotating channel. Proc. Indian Acad. Sci. 85: 383-401.

[10] Sivaprasad, R., Prasad Rao, D.R.V. and Krishna, D.V. (1988). Hall effects on unsteady MHD free and forced convection flow in a porous rotating channel. Indian $J$. pure appl. Math., 19(7): 688-696.

[11] Mittal, M. L. and Bhat, A. N. (1979). Forced convective heat transfer in a MHD channel with Hall and ion-slip currents, Appl. Sci. Res. 35(4): 251-264.

[12] Rao, D.R.V.P. and Krishna, D.V. (1982). Combined effect of free and forced convection on mhd flow in a rotating porous channel. Int. J. Math. Math. Sci. 5: 165-182

[13] Mori, Y. (1961). On combined free and forced convection laminar MHD flow and heat transfer in channel with transverse magnetic field. Int. Devel. Heat Transfer. 3: 1031.

[14] Yu, C. P. (1965): Combined forced and free convective channel in MHD, AIAA J., 3, 1184- 1185.

[15] Datta, N. and Jana, R. N. (1977). Hall effects on hydromagnetic convective flow through a channel with conducting walls. Int. J. Engng Sci. 15: 561-567.

[16] Ghosh, S. K. and Bhattacharjee, P. K. (2000). Magnetohydrodynamic convective flow in a rotating channel. Arch. Mech. 52(2): 303-318.

[17] Pop, I., Ghosh, S. K. and Nandi, D. K. (2001). Effects of the Hall current on free and forced convective flows in a rotating channel in the presence of an inclined magnetic field. Magnetohydrodynamics. 37(4): 348-359.

[18] Guria, M., Jana, R. N. (2007). Hall effects on the hydromagnetic convective flow through a rotating channel under general wall conditions, Magnetohydrodynamics. 43(3): $287-300$

[19] Guria. M., Das, B. K., Jana, R. N. and Ghosh, S. K. (2007).Effects of wall conductance on MHD fully developed flow with asymmetric heating of the wall. Int. J. Fluid Mech. Res. 34(6): 521-534.

[20] Seth, G.S. and Singh, M.K. (2008). Combined free and forced convection MHD flow in a rotating channel with perfectly conducting walls. Ind. J. Theo. Phys. 56: 203.

[21] Singha, K.G. (2009). Analytical solution to the problem of MHD free convective flow of an electrically conducting fluid between two heated parallel plates in the presence of an induced magnetic field. Int. J. Appl. Math. 
Computation. 1(4): 183-193.

[22] Seth, G. S., Mahto, N., Ansari, Md. S. and Nandkeolyar, R (2010).Combined free and forced convection flow in a rotating channel with arbitrary conducting walls. Int. J. Eng. Sci. Tech. 2(5), 184-197.

[23] Ahmed, S. (2010).Free and forced convective MHD oscillatory flow over an infinite porous surface in an oscillating free stream. Latin American Appl. Res. 40: 167-
173.

[24] Singh, K. D. (2012).Exact solution of MHD mixed convection periodic flow in a rotating vertical channel with heat radiation. Int. J. Phys. Math. Sci. 3(1): 14-30.

[25] Seth, G.S., Mahato, G.K. and Singh, J.K.(2013).Combined free and forced convection Couette-Hartmann flow in a rotating system with Hall effects. J. Nature Science and Sustainable Technology. 6(3):125-150. 